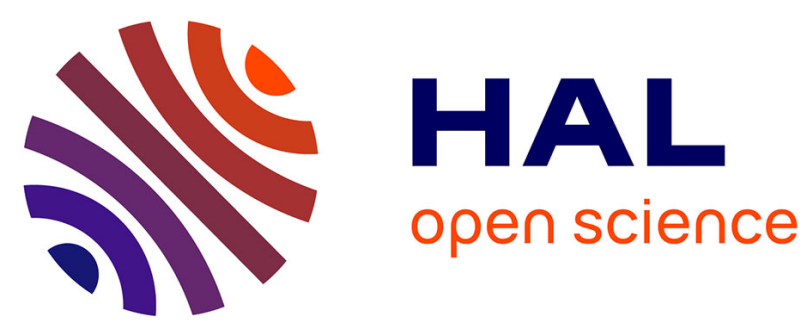

\title{
GLRT-Based Framework for the Multidimensional Statistical Resolution Limit
}

Mohammed Nabil El Korso, Remy Boyer, Alexandre Renaux, Sylvie Marcos

\section{To cite this version:}

Mohammed Nabil El Korso, Remy Boyer, Alexandre Renaux, Sylvie Marcos. GLRT-Based Framework for the Multidimensional Statistical Resolution Limit. IEEE International Workshop on Statistical Signal Processing 2011 - IEEE SSP'11, 2011, France. hal-00577273

\section{HAL Id: hal-00577273 https://hal.science/hal-00577273}

Submitted on 17 Mar 2011

HAL is a multi-disciplinary open access archive for the deposit and dissemination of scientific research documents, whether they are published or not. The documents may come from teaching and research institutions in France or abroad, or from public or private research centers.
L'archive ouverte pluridisciplinaire HAL, est destinée au dépôt et à la diffusion de documents scientifiques de niveau recherche, publiés ou non, émanant des établissements d'enseignement et de recherche français ou étrangers, des laboratoires publics ou privés. 


\title{
A GLRT-BASED FRAMEWORK FOR THE MULTIDIMENSIONAL STATISTICAL RESOLUTION LIMIT
}

\author{
Laboratoire des Signaux et Systèmes (L2S) \\ Université Paris-Sud XI, CNRS, SUPELEC \\ Gif-Sur-Yvette, France
}

Mohammed Nabil El Korso, Rémy Boyer, Alexandre Renaux and Sylvie Marcos

\begin{abstract}
Recently, a criterion for Multidimensional Statistical Resolution Limit (MSRL) evaluation, which is defined as the minimal separation to resolve two closely spaced signals depending on several parameters, was empirically proposed in [1] but without a statistical analysis. In this paper, we fill this lack by demonstrating that this MSRL criterion is asymptotically equivalent (upon to a translator factor) to a UMP (Uniformly Most Powerful) test among all invariant statistical tests. This result is an extension of a previous work on mono-dimensional SRL (i.e., when the signals only depend on one parameter). As an illustrative example, the 3-D harmonic retrieval case for wireless channel sounding is treated to show the good agreement of the proposed result.
\end{abstract}

\section{INDEX TERMS ${ }^{1}$}

Multidimensional statistical resolution limit, Cramér-Rao bound, uniformly most powerful test, performance analysis.

\section{INTRODUCTION}

Characterizing the ability of resolving closely spaced signals is an important step to quantify performance in array signal processing. The concept of Statistical Resolution Limit (SRL), i.e., the minimum distance between two closely spaced signals that allows a correct resolvability, is rising in several applications (especially in parameter estimation problems such as radar, sonar, spectral estimation [2], etc.)

It is important to note that, in several estimation problems, the signals are parameterized by more than one parameters of interest per signal, for example, in the context of, near-field source localization (bearing, elevation and range), polarized source localization (DOA and the polarization state parameters) and more generally in communication applications. However, the SRL has only been defined/derived in the mono-dimensional case [3-7] (i.e., for only one parameter of interest per signal.) This is way we recently have proposed an intuitive extension of the SRL for the multidimensional case [1], called the Multidimensional Statistical Resolution Limit (MSRL). This criterion is based on the extension of the so-called Smith criterion [5] (i.e., based on a Cramér-Rao Bound (CRB) approach) using the $k$-norm distance.

Nevertheless, no analysis on the MSRL (which was proposed in [1]) was done to check its behavior. Thus, the aim of this paper is to fill this lack. First, we link the concept of the MSRL to a binary hypothesis test which is a slight extension of [4].Second, we

\footnotetext{
${ }^{1}$ This project is funded by region Île de France and Digiteo Research Park.
}

prove that the MSRL criterion [1] is asymptotically equivalent to a Uniformly Most Powerful (UMP) test among all invariant statistical tests (which is, in the asymptotic case, considered as the strongest statement of optimality that one could expect to obtain [8]). Note that, while the aforementioned equivalence is derived in the context of 1-norm distance, the same conclusion can be extended for the MSRL based on other $k$-norm distances ( $k \geq 2$ with $k$ integer). Finally, we illustrate our result in the case of a 3-D harmonic retrieval model for wireless channel sounding.

\section{PROBLEM SETUP AND BACKGROUND}

Let $\left\{\Omega, \mathcal{F}, \mathbf{y}, \mathcal{Y}, \mathcal{A}, \mathcal{P}_{\boldsymbol{\xi}}, \boldsymbol{\xi} \subseteq \mathbb{R}^{2 P+Q}\right\}$ be the statistical experiment generated by a random vector $\mathbf{y}$ taking values on the measurable space $\{\mathcal{Y}, \mathcal{A}\}$. An observation $\mathbf{y}(\omega)$ is the realization of the random vector $\mathbf{y}$ where $\omega$ takes its value on the measurable space $\{\Omega, \mathcal{F}\}$. The distribution of $\mathbf{y}$ is assumed to belong to a family of probability measures $\mathcal{P}_{\boldsymbol{\xi}}$ on $\mathcal{Y}$ and parameterized by the vector $\boldsymbol{\xi} \subseteq \mathbb{R}^{2 P+Q}$. It is also assumed that $\mathcal{P}_{\boldsymbol{\xi}}$ is absolutely continuous w.r.t. a $\sigma$-finite positive measure $\mu$ on $\mathcal{Y}$, such that the Radon-Nikodym derivative (i.e., the likelihood function) $p(\mathbf{y} \mid \boldsymbol{\xi}) \triangleq d \mathcal{P}_{\boldsymbol{\xi}}(\mathbf{y}) / d \mu(\mathbf{y}), \forall \boldsymbol{\xi} \subseteq \mathbb{R}^{2 P+Q}$ exists. More precisely, the observation model is assumed to be structured as follows

$$
\mathbf{y}=\boldsymbol{f}\left(\boldsymbol{\xi}_{1}\right)+\boldsymbol{f}\left(\boldsymbol{\xi}_{2}\right)+\mathbf{n}
$$

where, from a signal processing point of view, $\mathbf{y} \in \mathbb{R}^{N}$ and $\mathbf{n} \in \mathbb{R}^{N}$ denote the noisy received data ${ }^{2}$ and the additive noise with a known probability density function (pdf). The noiseless received data are assumed to be the sum of two signals $\mathbf{f}\left(\boldsymbol{\xi}_{m}\right), m=1,2$ each one modelled from the same deterministic known waveform $\mathbf{f}($.$) param-$ eterized by a set of deterministic unknown vectors $\boldsymbol{\xi}_{m} \subseteq \mathbb{R}^{P+q_{m}}$, $m=1,2$ in which $q_{1}+q_{2}=Q$. The function $\mathbf{f}($.$) is assumed to$ be measurable and the model (1) is assumed to be parameter identifiable (i.e., the Fisher information matrix considered through this paper is non singular). All the parameters are collected in $\boldsymbol{\xi}=$ $\left[\boldsymbol{\xi}_{1}^{T} \boldsymbol{\xi}_{2}^{T}\right]^{T} \subseteq \mathbb{R}^{2 P+Q}$. With a proper rearrangement ${ }^{3}$ of $\boldsymbol{\xi}$ one obtains $\boldsymbol{\xi}=\left[\boldsymbol{\omega}^{T} \boldsymbol{\rho}^{T}\right]^{T}$ where $\boldsymbol{\omega} \subseteq \mathbb{R}^{2 P}$ and $\boldsymbol{\rho} \subseteq \mathbb{R}^{Q}$ denote, respectively, the parameter vector of interest, and the unwanted or nuisance parameters vector. This means that we consider $P$ parameters of interest for each signal. To the best of our knowledge, the result of the literature [5] on the SRL have been only proposed in the case of

\footnotetext{
${ }^{2}$ Let us note that the study where the observation are complex can be handled by the real model (1) by stacking the real and imaginary parts of the observation vector.

${ }^{3}$ To avoid a complication of notation, $\boldsymbol{\xi}$ denote both the vector parameter before and after the rearrangement. In the following we will only use the vector parameter after the rearrangement.
} 
one parameter of interest per signal. Nevertheless, the problem of deriving the so-called Multidimensional Statistical Resolution Limit (MSRL) based on the Cramér-Rao Bound (CRB) in the case of more than one parameter of interest per signal was recently studied in [1]. The assumptions used for the MSRL derivation are the following:

- A1. The element of the $\boldsymbol{\omega}$ are of the same nature, i.e., the parameters of interest have the same unit measurement. If no, please refer to [1, Remark 2] to overcome this assumption.

- A2. Each parameter of interest w.r.t. to the first signal, $\omega_{1}^{(p)}$, can be as close as possible to the parameter of interest w.r.t. to the second signal $\omega_{2}^{(p)}$, but not equal. This is not really a restrictive assumptions, since in most applications, having two or more identical parameters of interest is a zero probability event [9, p74]. Nevertheless, in the case where it exists $p$ such that $\omega_{1}^{(p)}=\omega_{2}^{(p)}$, please refer to [1, Subsection 3.1] to overcome this assumption.

Under these assumptions, the intuitive MSRL criterion is defined in [1] as follows:

Criterion 1 The MSRL, denoted by $\delta$, for the model (1) in the case of two signals and $P$ parameters of interest per signal is given as the implicit solution of the following equation

$$
\delta^{2}=C R B(\delta)
$$

where

$$
\delta=\sum_{p=1}^{P} \delta_{p}
$$

in which the so-called local SRLs are given by $\delta_{p} \triangleq\left|\omega_{2}^{(p)}-\omega_{1}^{(p)}\right|$ and where $C R B(\delta)$ denotes the $C R B$ w.r.t. $\delta$ for the observation model (1).

In the following the latter MSRL criterion is shown to be asymptotically equivalent (upon to a translator factor) to a UMP test among all invariant statistical tests.

\section{ANALYSIS OF THE MSRL}

\subsection{Hypothesis test formulation}

Resolving two closely spaced sources, with respect to their parameter of interest, can be formulated as a binary hypothesis test $[6,7,10]$. Let us consider the hypothesis $\mathcal{H}_{0}$ which represents the case where the two emitted signal sources are combined into one signal (i.e., $\forall p \in[1 \ldots P], \omega_{1}^{(p)}=\omega_{2}^{(p)}$, whereas the hypothesis $\mathcal{H}_{1}$ embodies the situation where the two signals are resolvable (i.e., $\exists p \in$ $[1 \ldots P]$, such that $\left.\omega_{1}^{(p)} \neq \omega_{2}^{(p)}\right)$. Consequently, one can formulate the hypothesis test as a simple one-sided binary hypothesis test as follows:

$$
\begin{cases}\mathcal{H}_{0}: & \delta_{\text {detection }}=0 \\ \mathcal{H}_{1}: & \delta_{\text {detection }}>0\end{cases}
$$

where $\delta_{\text {detection }}$ denotes a distance between two sets containing the parameters of interest. Let us denote these sets as $C_{1}$ and $C_{2}$ where $C_{q}=\left\{\omega_{q}^{(1)}, \omega_{q}^{(2)}, \ldots, \omega_{q}^{(P)}\right\}, q=1,2$. Consequently, $\delta_{\text {detection }}$ can be defined as

$$
\delta_{\text {detection }} \triangleq \sum_{p=1}^{P}\left|\omega_{2}^{(p)}-\omega_{1}^{(p)}\right| .
$$

Since the separation term $\delta_{\text {detection }}$ is unknown, it is impossible to design an optimal detector in the Neyman-Pearson sense. Alternatively, the Generalized Likelihood Ratio Test (GLRT) $[8,11]$ is a well known approach appropriate to solve such a problem. The GLRT is expressed as:

$$
\begin{aligned}
L_{G}(\mathbf{y}) & =\frac{\max _{\delta_{\text {detection }}, \boldsymbol{\rho}_{1}} p\left(\mathbf{y} \mid \delta_{\text {detection }}, \boldsymbol{\rho}_{1}, \mathcal{H}_{1}\right)}{\max _{\boldsymbol{\rho}_{0}} p\left(\mathbf{y} \mid \boldsymbol{\rho}_{0}, \mathcal{H}_{0}\right)} \\
& =\frac{p\left(\mathbf{y} \mid \hat{\delta}_{\text {detection }}, \hat{\boldsymbol{\rho}}_{1}, \mathcal{H}_{1}\right)}{p\left(\mathbf{y} \mid \hat{\boldsymbol{\rho}}_{0}, \mathcal{H}_{0}\right)} \underset{\mathcal{H}_{0}}{\stackrel{\mathcal{H}_{1}}{\gtrless} \varsigma^{\prime}}
\end{aligned}
$$

where $\hat{\delta}_{\text {detection, }} \hat{\boldsymbol{\rho}}_{1}$ and $\hat{\boldsymbol{\rho}}_{0}$ denote the Maximum Likelihood Estimator (MLE) of $\delta_{\text {detection }}$ under $\mathcal{H}_{1}$, the MLE of $\rho$ under $\mathcal{H}_{1}$ and the MLE of $\rho$ under $\mathcal{H}_{0}$. $\varsigma^{\prime}$ denotes the test threshold. We rewrite (6) to obtain

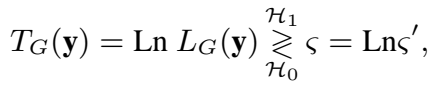

in which Ln denotes the natural logarithm.

\subsection{Asymptotic equivalence of the GLRT}

To find the analytical expression of $T_{G}(\mathbf{y})$ in (7) is generally intractable. This is mainly due to the fact that the derivation of $\hat{\delta}_{\text {detection }}$ is a highly non linear optimization problem [12] (aside from the linear parameter model $[8,13])$. Consequently, in the following, and as in [6], we consider the asymptotic case (in terms of number of samples). In [11, eq (6C.1)] it has been proved that, for a large number of snapshots, the statistic $T_{G}(\mathbf{y})$ follows a chi-squared pdf under $\mathcal{H}_{0}$ and $\mathcal{H}_{1}$ given by

$$
T_{G}(\mathbf{y}) \sim \begin{cases}\chi_{1}^{2} & \text { under } \mathcal{H}_{0} \\ \chi_{1}^{\prime 2}\left(\kappa^{\prime}\left(P_{f a}, P_{d}\right)\right) & \text { under } \mathcal{H}_{1}\end{cases}
$$

where $\chi_{1}^{2}$ and $\chi_{1}^{\prime 2}\left(\kappa^{\prime}\left(P_{f a}, P_{d}\right)\right)$ denote the central chi-square and the noncentral chi-square pdf with one degree of freedom. $P_{f a}$ and $P_{d}$ are, respectively, the probability of false alarm and the probability of detection w.r.t. hypothesis test (4). Whereas, assuming that $\mathrm{CRB}\left(\delta_{\text {detection }}\right)$ exist, the the noncentral parameter $\kappa^{\prime}\left(P_{f a}, P_{d}\right)$ is given by [11, p.239]

$$
\kappa^{\prime}\left(P_{f a}, P_{d}\right)=\delta_{\text {detection }}^{2}\left(\mathrm{CRB}\left(\delta_{\text {detection }}\right)\right)^{-1} .
$$

On the other hand, one can notice that the noncentral parameter $\kappa^{\prime}\left(P_{f a}, P_{d}\right)$ can be determined exclusively by the choice of $P_{f a}$ and $P_{d}[6,8]$ as the solution of

$$
\mathcal{Q}_{\chi_{1}^{2}}^{-1}\left(P_{f a}\right)=\mathcal{Q}_{\chi_{1}^{2}\left(\kappa^{\prime}\left(P_{f a}, P_{d}\right)\right)}^{-1}\left(P_{d}\right)
$$

in which $\mathcal{Q}_{\chi_{1}^{2}}^{-1}$ and $\mathcal{Q}_{\chi^{\prime}{ }_{1}^{\prime}\left(\kappa^{\prime}\left(P_{f a}, P_{d}\right)\right)}^{-1}$ are the inverse of the right tail of the $\chi_{1}^{2}$ and $\chi_{1}^{\prime 2}\left(\kappa^{\prime}\left(P_{f a}, P_{d}\right)\right)$ pdf.

Finally, (9) and (10) leads to

$$
\delta_{\text {detection }}=\kappa\left(P_{f a}, P_{d}\right) \sqrt{\mathrm{CRB}\left(\delta_{\text {detection }}\right)}
$$

where $\sqrt{\kappa\left(P_{f a}, P_{d}\right)}=\kappa^{\prime}\left(P_{f a}, P_{d}\right)$ is the so-called translation factor which is determined for a given probability of false alarm and probability of detection (see Fig. 1). 


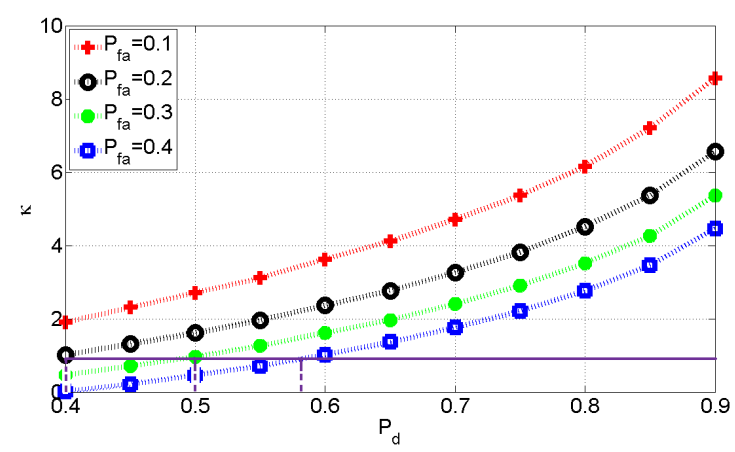

Fig. 1. The translator factor $\kappa$ vs. the probability of detection $P_{d}$ and $P_{f a}$. One can notice that increasing $P_{d}$ or decreasing $P_{f a}$ has the effect to increase the value of the translator factor $\kappa$. This is expected since increasing $P_{d}$ or decreasing $P_{f a}$ leads to a more selective decision $[8,11]$.

Remark 1 It is worth noting that, the hypothesis test (4) is a binary one-sided test and that the MLE used is an unconstrained estimator. Thus, one can deduce that the GLRT, used to derive the asymptotic $S R L$, is [6, 11]: i) the asymptotically uniformly most powerful test among all invariant statistical tests, and ii) has an asymptotic Constant False-Alarm Rate (CFAR). This is, in the asymptotic case, considered as the strongest statement of optimality that one could hope to obtain [8].

Finally, from (2) and (11), one can state the following result:

Result 1 The asymptotic MSRL based on the empirical extension of the Smith criterion given in (2) is equivalent (upon to a translation factor) to the binary one-sided hypothesis test given in (4). Consequently, it is equivalent to an asymptotically uniformly most powerful test among all invariant statistical tests.

Remark 2 Consequently, one should note that the MSRL based on the Smith criterion is exactly equal to the MSRL based on the detection approach for all values of $P_{f a}$ and $P_{d}$ such that $\kappa\left(P_{f a}, P_{d}\right)=1$ (cf, Fig. 1).

\section{NUMERICAL EXAMPLE}

In this Section we present a numerical example of the MSRL applied to the 3-D Harmonic retrieval model for wireless channel sounding $[9,14]$. First we briefly introduce the considered model, then, we numerically derive its MSRL.

\subsection{Model setup}

The observation model can be written as [9]

$$
[\mathcal{Y}(t)]_{k, l, m}=[\mathcal{X}(t)]_{k, l, m}+[\mathcal{N}(t)]_{k, l, m}, \quad t=1, \ldots, T,
$$

for $k=1 \ldots K, l=1 \ldots L, m=1 \ldots M$, in which $K, L$ and $M$ denote the number of acquired data samples per channel, the number of receive antenna sensors and the number of transmit antenna sensors, respectively. $\mathcal{Y}(t), \mathcal{X}(t)$ and $\mathcal{N}(t)$ denote the noisy observation multiway array, the noiseless observation multiway array and the noise multiway array at the $t^{t h}$ snapshot, respectively. Whereas, the noiseless observation multiway array is given by [14]

$$
[\mathcal{X}(t)]_{k, l, m}=\sum_{q}^{2} s_{q}(t) e^{j \omega_{q}^{(1)} k} e^{j \omega_{q}^{(2)} l} e^{j \omega_{q}^{(3)} m},
$$

where the so-called electrical angles are given by

$$
\begin{aligned}
& \omega_{q}^{(1)} \triangleq \frac{-2 \pi}{K} \tau_{q}, \\
& \omega_{q}^{(2)} \triangleq \frac{-2 \pi d_{R}}{\lambda} \cos \left(\phi_{q}\right), \\
& \omega_{q}^{(3)} \triangleq \frac{-2 \pi d_{T}}{\lambda} \cos \left(\theta_{q}\right),
\end{aligned}
$$

and $\tau_{q}, \phi_{q}, \theta_{q}$ denote delay, direction of arrival, and direction of departure, of the the $q^{t h}$ multipath, respectively. $d_{R}, d_{T}$ and $\lambda$ are the inter-element spacings of the transmit and receive array and the carrier wavelength, respectively. $s_{q}(t)$ is the $q^{t h}$ complex amplitude path [9]. It can be proved that the 3-D harmonic retrieval model for wireless channel sounding follows a PARAFAC (PARallel FACtor) decomposition [15] given by

$$
\mathcal{X}(t)=\sum_{q=1}^{2} s_{q}(t)\left(\boldsymbol{a}\left(\omega_{q}^{(1)}\right) \circ \boldsymbol{a}\left(\omega_{q}^{(2)}\right) \circ \boldsymbol{a}\left(\omega_{q}^{(3)}\right)\right),
$$

where $\left[\boldsymbol{a}\left(\omega_{q}^{(p)}\right)\right]_{i}=e^{j \omega_{q}^{(p)}(i-1)}$ and $\circ$ denotes the multiway array outer-product [16]. After vectorization, the full noise free observation vector is given by

$$
\boldsymbol{x}=\left[\begin{array}{lll}
\operatorname{vec}^{T}(\mathcal{X}(1)) & \ldots & \operatorname{vec}^{T}(\mathcal{X}(L))
\end{array}\right]^{T} .
$$

In the same way, we define $\mathbf{y}$, the noisy observation vector, and $\mathbf{n}$, the noise vector, by the concatenation of the proper multiway array's entries.

\subsection{MSRL derivation}

First we derive the CRB for the 3-D Harmonic retrieval model. Then, we use the change of variable formula [13, p 45] to deduce $\operatorname{CRB}(\delta)$. Assuming i.i.d. complex circular white Gaussian noise with zeromean and unknown variance $\sigma^{2} \mathbf{I}$, the joint pdf of $\mathbf{y}$ for a given unknown deterministic parameter vector $\boldsymbol{\xi}$ is

$$
\begin{aligned}
p(\mathbf{y} \mid \boldsymbol{\xi}) & =\prod_{t=1}^{T} p(\operatorname{vec}(\mathcal{Y}(t)) \mid \boldsymbol{\xi}) \\
& =\frac{1}{\left(\pi \sigma^{2}\right)^{T L M K}} e^{\frac{-1}{\sigma^{2}}(\mathbf{y}-\mathbf{x})^{H}(\mathbf{y}-\mathbf{x})}
\end{aligned}
$$

The unknown parameter vector is given by

$$
\boldsymbol{\xi}=\left[\begin{array}{lllllll}
\omega_{1}^{(1)} & \omega_{1}^{(2)} & \omega_{1}^{(3)} & \omega_{1}^{(1)} & \omega_{1}^{(2)} & \omega_{1}^{(3)} & \sigma^{2}
\end{array}\right]^{T},
$$

where the parameters of interest are $\omega_{1}^{(1)}, \omega_{1}^{(2)}, \omega_{2}^{(1)}, \omega_{2}^{(2)}$ (representing the direction of arrival and the direction of departure of each sources). After some calculus, the Fisher information matrix of the noisy observations $\mathbf{y}$ for orthogonal known amplitudes, is given by

$$
\boldsymbol{F I M}(\boldsymbol{\xi})=\frac{2}{\sigma^{2}}\left[\begin{array}{cc}
\mathbf{F}_{\omega} & \mathbf{0} \\
\mathbf{0} & \times
\end{array}\right]
$$


where,

$$
\mathbf{F}_{\boldsymbol{\omega}}=T K M L(\boldsymbol{\Delta} \otimes \mathbf{G})
$$

in which

$$
\boldsymbol{\Delta}=\left[\begin{array}{cc}
\sum_{t=1}^{T}\left\|s_{1}(t)\right\|^{2} & 0 \\
0 & \sum_{t=1}^{T}\left\|s_{2}(t)\right\|^{2}
\end{array}\right]
$$

and

$$
\mathbf{G}=\left[\begin{array}{lll}
\frac{(2 K-1)(K-1)}{6} & \frac{(K-1)(L-1)}{2} & \frac{(K-1)(M-1)}{2} \\
\frac{(L-1)^{2}(K-1)}{2} & \frac{(2 L-1)^{2}(L-1)}{6} & \frac{(L-1)^{2}(K-1)}{2} \\
\frac{(M-1)}{2} & \frac{(M-1)(L-1)}{2} & \frac{(2 M-1)}{6}
\end{array}\right] .
$$

Now one can apply Criterion 1. From (15), one deduces numerically $\mathbf{C R B}(\boldsymbol{\xi})=(\mathbf{F I M}(\boldsymbol{\xi}))^{-1}$. Then, applying the change of variable formula [13, p 45], one obtains

$$
\operatorname{CRB}(\delta)=\sum_{p=1}^{2} \sum_{q=1}^{2} \operatorname{CRB}\left(\omega_{q}^{(p)}\right)+\sum_{q=1}^{2} \operatorname{CRB}\left(\omega_{q}^{(1)}, \omega_{q}^{(2)}\right)
$$

Finally, solving numerically the implicit equation $\delta^{2}=\mathrm{CRB}(\delta)$ gives the desired MSRL as reported in Fig. 2.

From Fig. 2 one can notice that the numerical MSRL based on Criterion 1 is in good agreement with the MSRL derived using the hypothesis test approach. One can notice that, for $P_{d}=0.37$ and $P_{f a}=0.1$ the MSRL based on Criterion 1 is exactly equal to the MSRL based on the hypothesis test derived in the asymptotic case. From the case $P_{d}=0.49$ and $P_{f a}=0.3$ and $P_{d}=0.32$ and $P_{f a}=0.1$, one can notice the influence of the multiplicative factor $\kappa$ on the MSRL.

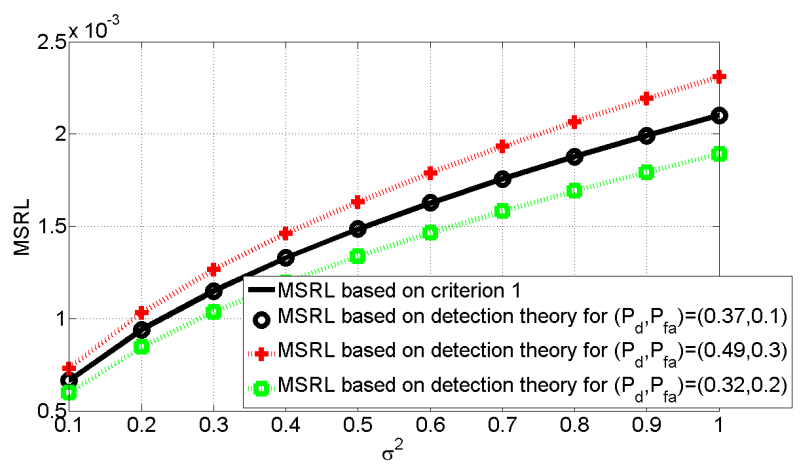

Fig. 2. MSRL vs. $\sigma^{2}$ for $L=100$ : one can notice that the MSRL based on the Criterion 1 is in good agreement with the MSRL based on the hypothesis test approach (which uses an asymptotically uniformly most powerful test among all invariant statistical tests).

\section{CONCLUSION}

In this paper, we have analyzed the multidimensional statistical resolution limit based on the empirical extension of the Smith criterion. More precisely, it has been demonstrated that the empirical MSRL criterion based on the 1-norm distance is asymptotically equivalent (upon to a translator factor) to a uniformly most powerful test which is (in the asymptotic case) considered as the strongest statement of optimality that one could expect to obtain.

\section{REFERENCES}

[1] M. N. El Korso, R. Boyer, A. Renaux, and S. Marcos, "Statistical resolution limit for multiple signals and parameters of interest," in Proc. of IEEE Int. Conf. Acoust., Speech, Signal Processing, Dallas, TX, 2010.

[2] H. L. VanTrees, Detection, Estimation and Modulation Theory. New York: Wiley, 1968, vol. 1.

[3] H. B. Lee, "The Cramér-Rao bound on frequency estimates of signals closely spaced in frequency," IEEE Trans. Signal Processing, vol. 40, no. 6, pp. 1507-1517, 1992.

[4] M. Shahram and P. Milanfar, "Imaging below the diffraction limit: A statistical analysis," IEEE Trans. Image Processing, vol. 13, no. 5, pp. 677-689, May 2004.

[5] S. T. Smith, "Statistical resolution limits and the complexified Cramér Rao bound," IEEE Trans. Signal Processing, vol. 53, pp. 1597-1609, May 2005.

[6] Z. Liu and A. Nehorai, "Statistical angular resolution limit for point sources," IEEE Trans. Signal Processing, vol. 55, no. 11, pp. 5521-5527, Nov. 2007.

[7] A. Amar and A. Weiss, "Fundamental limitations on the resolution of deterministic signals," IEEE Trans. Signal Processing, vol. 56, no. 11, pp. 5309-5318, Nov. 2008.

[8] L. L. Scharf, Statistical Signal Processing: Detection, Estimation, and Time Series Analysis. Reading: Addison Wesley, 1991.

[9] A. Gershman and N. Sidiropoulos, Space-time processing for MIMO communications. New York: Wiley, 2005.

[10] M. Shahram and P. Milanfar, "On the resolvability of sinusoids with nearby frequencies in the presence of noise," IEEE Trans. Signal Processing, vol. 53, no. 7, pp. 2579-2585, July 2005.

[11] S. M. Kay, Fundamentals of Statistical Signal Processing : Detection Theory. NJ: Prentice Hall, 1998, vol. 2.

[12] B. Ottersten, M. Viberg, P. Stoica, and A. Nehorai, "Exact and large sample maximum likelihood techniques for parameter estimation and detection in array processing," in Radar Array Processing, S. Haykin, J. Litva, and T. J. Shepherd, Eds. Berlin: Springer-Verlag, 1993, ch. 4, pp. 99-151.

[13] S. M. Kay, Fundamentals of Statistical Signal Processing. NJ: Prentice Hall, 1993, vol. 1.

[14] K. Mokios, N. Sidiropoulos, M. Pesavento, and C. Mecklenbrauker, "On 3-D harmonic retrieval for wireless channel sounding," in Proc. of IEEE Int. Conf. Acoust., Speech, Signal Processing, vol. 2, Philadelphia, U.S.A., 2004, pp. 89-92.

[15] R. Harshman, Foundations of the PARAFAC procedure: Models and conditions for an "explanatory" multi-modal factor analysis. UCLA Working Papers in Phonetics, 1970.

[16] G. H. Golub and C. F. V. Loan, Matrix Computations. London: Johns Hopkins, 1989. 